\title{
LPV VS MULTI-MODEL PI(D) GAIN-SCHEDULING APPLIED TO CANAL CONTROL ${ }^{*}$
}

\author{
Yolanda Bolea ${ }^{1}$, Vicenç Puig ${ }^{1}$, Joaquín Blesa ${ }^{1}$, Manuel Gómez ${ }^{2}$ and José Rodellar ${ }^{3}$ \\ ${ }^{1}$ Automatic Control Depart.(ESAII), ${ }^{2}$ Hydraulics Depart., ${ }^{3}$ Mathematical Depart. \\ Technical University of Catalonia (UPC)

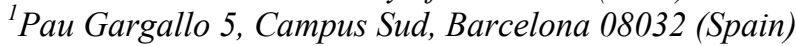 \\ yolanda.bolea@upc.es
}

\begin{abstract}
This paper compares the multi-model gain scheduling (GS) control versus the linear parameter varying (LPV) gain scheduling control. The results of this comparative study is carried out exclusively with PI controllers. Although the simplicity and easiness of the former approach for controlling non-linear industrial plants, its main drawback relies on the impossibility to assure stability and performance for slow parameter variation. On the other hand, the later approach rigorously ensures stability and performance of the control system for any variation (smooth as well as abrupt) of the plant parameters. Finally, both methodologies are tested on a test bench canal. Copyright (C) 2005 IFAC
\end{abstract}

Keywords: Multi-Model GS, LPV GS, PI(D) Controller, Nonlinear Systems, Environmental Systems.

\section{INTRODUCTION}

Nowadays, agricultural systems are receiving considerable interest because they are able to increase demand in water savings and minimise the losses. For these reasons, lastly, hydraulic and control engineers use automatic control techniques in order to obtain a better performance in real-time operation of open canal systems and to deliver water to the farmers on demand. So far, the main used control strategies have been classical PI(D) controllers (Chentouf,2001) and predictive strategies (Gomez,2002). For representing non-linear parameter distributed model dynamics with linear model parameter variation within the operating regime, multi-models or linear parameter varying (LPV) should be considered. In both cases, gain-scheduling (GS) control would be a suitable control strategy (Rugh,2000). There are two types of GS: Conventional or classical multi-model GS and Linear parameter-varying (LPV) GS. The classical GS consists in designing linear time-invariant (LTI) controllers for several operating points and then applying an interpolation strategy to obtain a global control. Consequently, powerful tools for linear systems can be applied to non-linear plants. In spite of the numerous GS applications, there is not a formal framework until the beginning of the nineties (Shamma,1990). This framework gives heuristic rules to ensure global stability and global performance, but it does not provide a systematic design procedure. Latter, in (Shamma, 1991), linear parameter-varying (LPV) systems are introduced. In this context, the synthesis problem can be formulated as a convex optimisation problem with linear matrix inequality (LMI) constraints wherein the controller is considered as a simple entity without the classical interpolation drawbacks (Becker, 1994; Apkarian,1995; Packard, 1996).

\footnotetext{
* This work is partially supported by contract REN 2002-00032 of Spanish Ministry of Science and Technology.
} 
Then, with the goal of preventing these drawbacks in control canal systems LPV GS control seems an interesting solution to be considered. In fact, lastly, it has been formulated an LPV model that reproduces more faithfully the non-linear and time-varying characteristics of a canal than the typical LTI models obtained using hydraulic laws (Schuurmans, 1995) or classical identification (Bolea,2002).

This work focus on the comparative analysis of both control GS techniques in case of using a PI law regarding its application to canals. So far, the differences between classical multi-model and LPV GS has been only been treated in the literature (Shamma,1991; Groot Wassink,2005) when advanced control methods are used, but, there are not any comparison when a simple controllers are used. PI(D) controllers are the usual control laws in real canal control application since their low order, easy implementation and physical significance.

The paper is organized as follows. A brief review of multi-models and LPV models and their associated control strategies is introduced in Section 2. In Section 3 both modelling methodologies are applied to a single pool canal while in Section 4 PI controllers are designed, tested and compared using both GS approaches. Finally, in Section 5, the conclusions (advantages and limitations of both techniques) of this comparative study are presented.

\section{MULTI-MODEL VERSUS LPV GS CONTROL}

\subsection{Conventional multi-model GS}

Conventional or classical multi-model GS is widely used technique to control non-linear systems in variety of engineering applications. The parameters ("gains") of the gain-scheduling controllers are typically chosen using LTI techniques using a two step process. First, several operating points are selected to cover the range of system dynamics. At each of these points, the designer obtain an LTI model that approximate the real plant and designs a linear controller for each one. This process gives a set of linear feedback control laws that performs satisfactorily when the closed-loop system is operated near the respective operating-points. A global nonlinear controller for the nonlinear system is then obtained by interpolation or 'scheduling' the "gains" from the local operating point designs. Therefore, this technique usually uses a grid of LTI models (Johansen, 1996; Tanaka,2002) obtained by physical laws or classical identification (Ljung,1999). A well-known limitation of this methodology is due to the fact that only slowly varying trajectories are admissible for the controller in order to assure stability and desired performance in closed-loop.

The controller design procedure is based on the representation of a given nonlinear plant in terms of the multi-model given by:

$$
\begin{gathered}
R_{i}: \text { IF } \theta_{1}(t) \text { is } M_{1 i} \text {, and } \ldots \text { and } \theta_{j}(t) \text { is } M_{j i} \\
\dot{x}_{i}(t)=A_{i} x(t)+B_{i} u(t) \\
y_{i}(t)=C_{i} x(t)+D_{i} u(t) .
\end{gathered}
$$

The antecedent part of each rule $R_{i}$ contains fuzzy or multi-model linguistic descriptions $M_{j i}$, of the scheduling variables $\theta_{j}(t)$ and the consequent part contains a local linear model of the nonlinear system. The entire multi-model of the plant (1) is obtained by fuzzy blending of the consequent local models. For a given pair of vectors $x(t)$ and $u(t)$, the final output of the global system is inferred as weighted sum of the contributing local models

$$
\begin{aligned}
\dot{x}(t) & =\frac{\sum_{i=1}^{r} \omega_{i}(\theta(t))\left[A_{i} x(t)+B_{i} u(t)\right]}{\sum_{i=1}^{r} \omega_{i}(\theta(t))} \\
y(t) & =\frac{\sum_{i=1}^{r} \omega_{i}(\theta(t))\left[C_{i} x(t)+D_{i} u(t)\right]}{\sum_{i=1}^{r} \omega_{i}(\theta(t))}
\end{aligned}
$$

with $\quad \omega_{\mathrm{i}}(\theta(t))=\operatorname{aggop}\left[M_{1 i}\left(\theta_{1}(t)\right) \ldots . . M_{j i}\left(\theta_{j}(t)\right)\right]$ where $\omega_{i}(\theta(t)) \geq 0$ is the degree of fulfilment of rule $i$, $\operatorname{aggop}(\cdot)$ is the aggregation operator (for instance, the product or the minimum), $\sum_{i=1}^{r} \omega_{i}(\theta(t))>0$ for all $i=1,2, \ldots, r$.

With $0 \leq \lambda_{i}(\theta(t))=\left(\omega_{i}(\theta(t)) / \sum_{j=1}^{r} \omega_{j}(\theta(t))\right) \leq 1$, (2) and (3) can be written as

$$
\begin{aligned}
\dot{x}(t) & =\sum_{i=1}^{r} \lambda_{i}(\theta(t))\left[A_{i} x(t)+B_{i} u(t)\right] \\
y(t) & =\sum_{i=1}^{r} \lambda_{i}(\theta(t))\left[C_{i} x(t)+D_{i} u(t)\right] .
\end{aligned}
$$

There are different inference weights to combine the obtained LTI controllers (Johansen,1996; Tanaka,2002). In the continuous-time case, the simplest fuzzy control or classical GS rule being considered here, has the form:

$R_{i}$ : IF $\theta_{1}(t)$ is $M_{1 i}$, and $\ldots$ and $\theta_{j}(t)$ is $M_{j i}$, then $u_{i}(t)=-F_{i} x(t)$

where $F_{i}$ is the controller matrix. The controller's output is inferred as the weighted mean

$$
u(t)=\frac{\sum_{i=1}^{r} \omega_{i}(\theta(t))\left(-F_{i} x(t)\right)}{\sum_{i=1}^{r} \omega_{i}(\theta(t))}
$$

which yields

$$
u(t)=\sum_{i=1}^{r} \lambda_{i}(\theta(t))\left(-F_{i} x(t)\right)=-F(\theta(t)) x(t) .
$$

If the scheduling vector $\theta(t)$ is a function of the state vector $x(t), u(t)$ represents a non-linear gainscheduling control law. 
The goal of the controller design is to determinate the constant matrix $F_{i}$ such that the desired dynamics of the closed-loop system and some desired steady-state input-output behaviour are obtained. For this purpose any classical design methods as pole placement, optimal control, etc. can be used.

\subsection{LPV Control}

To solve the main drawback of classical multi-model GS methodology, LPV GS provides a rigorous methodology that assure the stability and desired performance for any variation (smooth or abrupt) of the system parameters. But, to use this method it is necessary to formulate a LPV model by physical laws $(\mathrm{Wu}, 1995)$ or experimental identification (Bamieh,2002).

LPV systems are defined as linear systems whose dynamics depend on exogenous time-varying signals. When the variation of the parameters depends on the state space variables and/or input variables, they are denoted quasi-LPV or pseudo-LPV. In this case system dynamics can be represented as (Rugh,2000)

$$
\begin{aligned}
& \dot{x}(t)=A(\theta(t)) x(t)+B(\theta(t)) u(t) \\
& y(t)=C(\theta(t)) x(t)+D(\theta(t)) u(t) .
\end{aligned}
$$

where the system model is linear in both $x(t)$ and $u(t)$ but whose matrices $A(\cdot) \ldots D(\cdot)$ are not constant but variable. The LPV models describes how the dynamics of a system vary as a function of one or more scheduling parameters $\theta(t)$ that itself can depend on time.

LPV based gain-scheduling techniques are replacing classical gain-scheduling techniques and becoming more widely used in control design. In ( $\mathrm{Yu}, 2002$; Groot Wassink,2005) are presented design examples and applications. Once an LPV model is built, several control synthesis techniques can be used for controller design, LQG control (Wu, 1995), $H_{\infty}$ control (Apkarian,1995), predictive control (Goto,2003), etc. Many of the control system design techniques using LPV models can be cast or recast as convex problems that involve linear matrix inequalities (LMI).

If the original plant (9) is polytopic verifying condition quadratic $H_{\infty}$ performance and/or LMI region (pole-placement method), etc. only on the vertices (Vertex Property) of the parameter hyperrectangle $\Theta$ is necessary and sufficient for verifying such a condition for all parameters $\theta \in \Theta(\mathrm{Yu}, 2002)$. This implies that the number of inequalities needed to test the design conditions can be reduced to a finite one. Then, the model of the dynamics of the LPV system (9) can be represented

$$
G(s, \theta(t)):=\left[\begin{array}{ll}
A(\theta(t)) & B(\theta(t)) \\
C(\theta(t)) & D(\theta(t))
\end{array}\right]=\sum_{i=1}^{r} \lambda_{i}\left[\begin{array}{ll}
A_{i} & B_{i} \\
C_{i} & D_{i}
\end{array}\right]
$$

with $\theta(t) \in \Theta:=C o\left\{v_{1}, v_{2}, \ldots, v_{r}\right\}=\left\{\sum_{i=1}^{r} \lambda_{i} v_{i}: \lambda_{i}>0, \sum_{i=1}^{r} \lambda_{i}=I\right\}$

where $v_{i}, i=1, \ldots, r$ are the vertices of the polytope approximating the exact parameter space.
Then, the following design problem is outlined: to obtain a static controller, $F$ of the following way

$$
\begin{aligned}
& \dot{x}_{c l}=A_{c l}(\theta) x_{c l}+B w \\
& y=C_{c l}(\theta) x_{c l}+D w
\end{aligned}
$$

which guarantees difference conditions (of stability and performance) (Apkarian,1995; Yu,2002) for any variation of the parameters. Since the plant is assumed polytopic, it can be realised a polytopic design of the feedback controller $F(\theta)$ such as

$$
u(t)=-F(\theta(t)) x(t)(12)
$$

where

$$
\begin{gathered}
F(\theta) \in \operatorname{Co}\left\{F\left(v_{1}\right), F\left(v_{2}\right), \ldots, F\left(v_{r}\right)\right\}:=\left\{\sum_{i=1}^{r} \lambda_{i} F_{i} ; F_{i}=F\left(v_{i}\right)\right\} \\
F(\theta)=\lambda_{1} F\left(v_{1}\right)+\lambda_{2} F\left(v_{2}\right)+\ldots+\lambda_{r} F\left(v_{r}\right) \text { where } \\
\sum_{i=1}^{r} \lambda_{i}(\theta)=1, \lambda_{i}(\theta)>0
\end{gathered}
$$

This technique is known as convex decomposition technique, and $C o$ is the function that forms a convex hull from the polytope vertices. The polytopic coordinates are calculated by fast algorithms in such a way that for each vertex $v_{i}, i=1, \ldots, r$ its coordinates are

$$
\begin{aligned}
& \lambda_{i}=\prod_{j=1}^{l} \tilde{\vartheta}_{j}^{i}, \quad \text { with }
\end{aligned}
$$

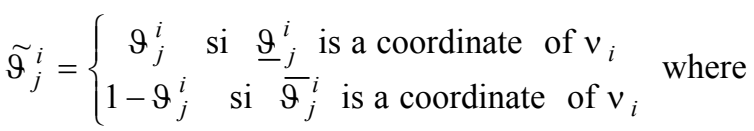

$$
\begin{aligned}
& \tilde{\vartheta}_{j}^{i}=\frac{\left(\bar{\theta}_{j}^{i}-\theta_{j}^{i}\right)}{\left(\bar{\theta}_{j}^{i}-\underline{\theta}_{j}^{i}\right)}, j=1, \ldots, l .
\end{aligned}
$$

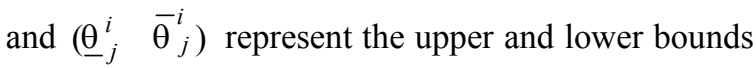
of $\theta_{j}^{i}$, and $l$ fulfils that $r=2^{l}$.

Then, the matrices $A_{c l}(\theta)$ and $C_{c l}(\theta)$ that depends on the parameter vector $\theta$ can be expressed by

$$
A_{c l}=\left\{\sum_{i=1}^{r} \lambda_{i}(\theta)\left(A_{i}+B F_{i}\right)\right\}, B_{c l}=\left\{\sum_{i=1}^{r} \lambda_{i}(\theta)\left(C+D F_{i}\right)\right\} \text {. }
$$

As a major advantage, LPV gain-scheduling offers a systematic design approach for guaranteeing stability and performance over the whole operating regime. Unfortunately, the design cycle is strongly affected by issues of LPV modeling and numerical conditioning. Moreover, algorithms that are practically applicable suffer from conservatism caused by the underlying assumption that the scheduling-parameters can change arbitrarily fast in time. Consequently, the number of actual industrially relevant implementation of LPV control is rather restricted (Groot Wassink,2005).

\subsection{Connection between both types of techniques}

The multi-model (1) can also be regarded as a quasilinear varying system, i.e., system linear in both $x(t)$ and $u(t)$ whose matrices $A(\cdot) \ldots . . D(\cdot)$ are not constant, 
but varying as (9). From (4) and (5), one can see that for all possible values of $\theta(t)$, which are assumed to be known on-line, these matrices are bounded within a polytope whose vertices are the matrices of the individual rules as in (10). Therefore, in the same way we can develop the control law from (7) and (8) to (12) and (13). Notice that in case of an LPV model both control techniques can be used. But, in case of multi-models LPV techniques can not be applied.

\section{MODELLING OF A CANAL: MULTI-MODEL VERSUS LPV APPROACH}

\subsection{Description of the system}

The complete water behaviour is accurately reproduced by Saint-Venants's equations using a simulator developed by the group of "Modelling and Control of Hydraulic Systems" at the UPC. All the experiments presented in this paper will be done on this simulator. This canal is composed by a single pool equipped with an upstream sluice gate and a downstream spillway (Fig.1). Upstream of this gate there is a damming of constant level $H=3.5 \mathrm{~m}$. The total length of the pool is $L=2 \mathrm{~km}$, with an initial flow $Q_{0}=1 \mathrm{~m}^{3} / \mathrm{s}$, a gate discharge coefficient $C_{d g}=0.6$, a Manning roughness coefficient $n=0.014$, gate width and canal width $B=2.5 \mathrm{~m}$, a downstream spillway of height $y_{s}=0.7 \mathrm{~m}$, a spillway coefficient $C_{d s}=2.66$, and a bottom slope $I_{0}=5 \cdot 10^{-4}$.
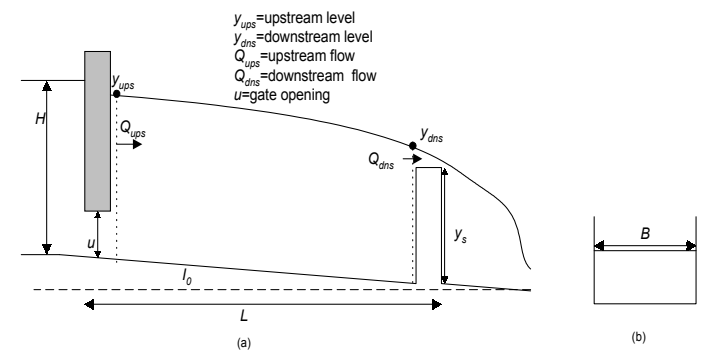

Fig.1. Canal scheme.(a) Longitudinal and (b) cross section.

\subsection{Multi-model}

In order to design the multi-model GS controller four operating points have been chosen. They correspond to the four gate opening positions $(j=1,2,3,4)$, which are representative of four operating modes: low, lowmedium, high-medium, and high water flow rate. The dynamic study consists of obtaining the step response around each operating point. A response analysis show that the dynamics (see Fig.2) can be simply approximated by a first-order system with time delay (FOPDT) as follows

$$
G_{j}(s)=\frac{k_{j} e^{-\tau} s}{T_{j} s+1}
$$

where $k_{j}$ is the $j^{\text {th }}$ static gain, $T_{j}$ is the $j^{\text {th }}$ dominant time constant and $\tau_{j}$ is the $j^{\text {th }}$ time delay.

This decomposition of operating range $(0.9 \leq u \leq 0.1$ $[\mathrm{m}])$ is selected such that the time constant varies with less than 1.2 between two neighbouring regimes. Thus, assuming the local models are exactly correct at the centre points of their corresponding regimes, the interpolated model time constant are never more than $\sqrt{1.2}$ of error. The weighting functions used in the interpolation are presented in Fig.3. They are designed to give a smooth transition between the operating regimes.

Table 1. Continuous transfer function parameters with respect to the gate position $j$

\begin{tabular}{cccc} 
OPERATING & \multicolumn{3}{c}{ PARAMETERS } \\
POINT $j$ & $K_{i}$ & $T_{i}[\mathrm{sec}]$ & $\tau_{i}[\mathrm{sec}]$ \\
\hline 1 & 1.43 & 788.02 & 496.80 \\
2 & 0.96 & 612.74 & 416.40 \\
3 & 0.73 & 546.45 & 376.20 \\
4 & 0.58 & 457.25 & 351.60 \\
\hline
\end{tabular}
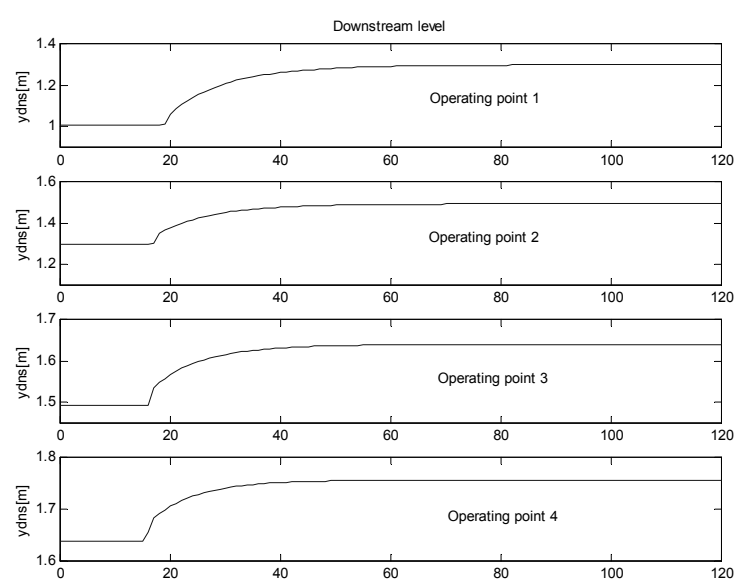

Fig.2. Step time responses for the four operating points: 1 ( $u$ from 0.1 to $0.3 \mathrm{~m}$ ), 2 ( $u$ from 0.3 to $0.5 \mathrm{~m}$ ), 3 ( $u$ from 0.5 to $0.7 \mathrm{~m}$ ) and 4 ( $u$ from 0.7 to $0.9 \mathrm{~m}$ ).

The classical identification (for more details, see (Ljung,1999, Bolea,2002) of these three parameters with respect to the gate opening $j$ gives the values summarized in Table 1. An increase in the upstream water flow, i.e., an increase of gate opening $j$, implies a decrease in the gain $k_{j}$ and in the time constant $T_{j}$ as well as in the time delay $\tau_{j}$.

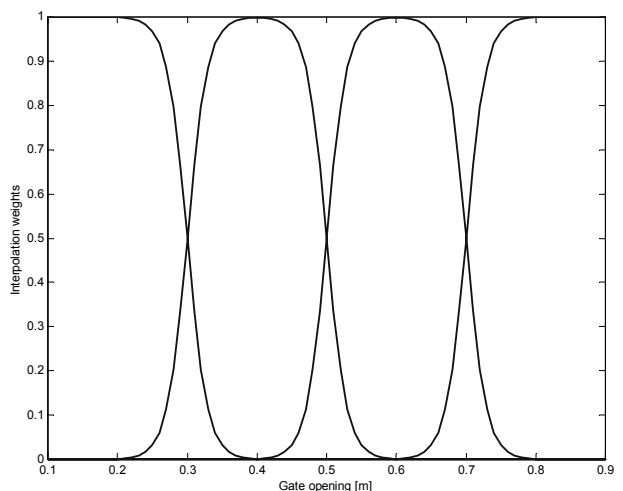

Fig.3. Weighting functions used in the gain-scheduler.

\subsection{LPV model}

In case of the irrigation canal, the plant dynamics depends on the upstream gate opening $(u)$. Then, it is interesting for control purposes to dispose of an 
approximate model which describes the non-linearity and time-varying parameter with the operating points. For these reasons in the literature an LPV model that takes account all these properties is proposed. This model is exhaustively presented and explained in (Bolea,2004). In this case the quasi-LPV model has a FOPDT structure, too

$$
G(s, u)=\frac{k(u)}{T(u) s+1} e^{-\tau(u) s}
$$

where the parameters (theoretical steady-state gain, $k(u)$; time constant $T(u)$, and delay vary with the gate opening, i.e. with the operating mode).

The expressions of these previous parameters (derived from hydraulic laws) are (for more details, see (Bolea,2004))

- Theoretical-state gain

$k(u)=\left(0.110^{-9}(b(46 \sqrt{u}-3))^{1 / 9}\left(\sqrt{I_{0}} / n C_{d s} B\right)^{2 / 3} b\left(0.9210^{10} b\right.\right.$

$\left.\left.+0.2510^{11} \sqrt{u}-0.1610^{10}\right)\right) /\left(\sqrt{u}(b+4.60 \sqrt{u}-0.3)^{13 / 9}\right)$

- Time delay

$$
\hat{\tau}(u)=\frac{k_{1}}{\left(1+k_{2} u\right)} \approx k_{1} e^{-k_{2} u}
$$

where:

$k_{1}=2 L /\left(f_{2}\left(y_{d n s}\right)+2 \hat{c}(\hat{y})\right), k_{2}=f_{1}\left(y_{u p s}\right) /\left(f_{2}\left(y_{d n s}\right)+2 \hat{c}(\hat{y})\right)$

$f_{1}\left(y_{\text {ups }}\right)=C_{d g} \sqrt{2 g} \sqrt{H-y_{u p s}} / y_{u p s}, f_{2}\left(y_{d n s}\right)=C_{d s}\left(y_{d n s}-y_{s}\right)^{3 / 2} / y_{d n s}$

The approximation of the expression (18) is based on Taylor series of first order: $e^{a u} \approx(1+a u)$ when $\mathrm{au} \rightarrow 0$.

- Time constant

$$
T(u)=\kappa\left[\frac{L}{v+c}\right] \quad \kappa \in[1.32,1.58]
$$

The nominal time constant, $T_{\text {nom }}(u)$, is approximated by $T_{\text {nom }}(u) \approx 1.45 \tau(u)=1.45 k_{1} e^{-k_{2} u}$.

\section{RESULTS OF CONTROL COMPARATIVE}

To achieve comparable results between the control methodologies proposed in Section 2, in the multimodel GS PI pole placement method (Åström,1997) is used in order to calculate the controller at each operating point, then a test of quadratic stability by LMIs (Tanaka,2001) is applied to guarantee the stability for any variation of the parameters inside the parameters space. For polytopic LPV GS PI (Bolea,2005) the quadratic $H_{\infty}$ performance condition through the Quadratic Real Lemma is used to guarantee quadratic stability, control system bandwidth and bounded control signal, and closedloop pole placement requirements by LMI regions (Yu,2002).

The delay is handled using a Predictor Smith configuration, and the theoretical steady-state gain is compensated by a inverse factor $k^{-1}$ ( $\AA$ ström,1997).

The control objective is to satisfy the water demand from farmers (i.e. the discharge $y_{d n s}$ ). The desired step response must fulfil the following specifications: a damping factor of $\zeta \geq 0.5$ and a bandwidth $B W \leq 0.0025 \mathrm{rad} / \mathrm{sec}$.

The multi-model GS is obtained by the combination of the PI controllers obtained at each operating point using the interpolation weights presented in the Fig.3.

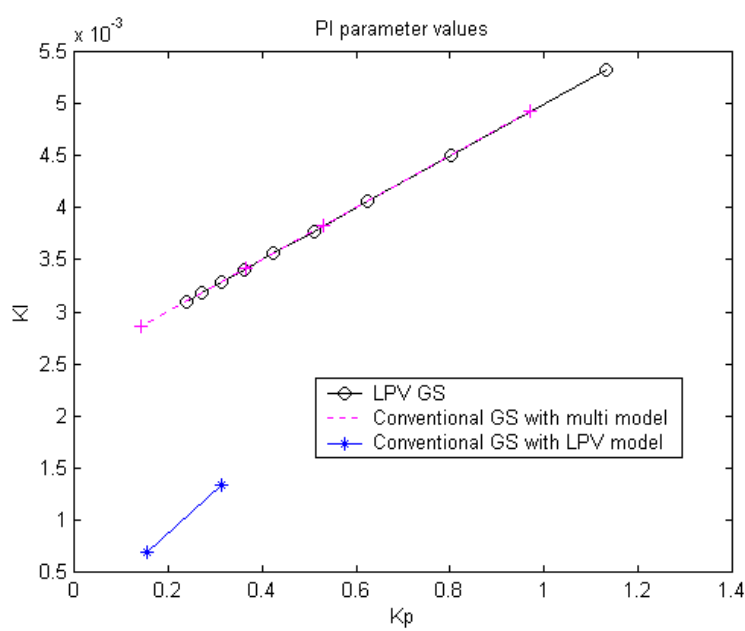

Fig.4. PI parameter values for: classical GS at each operating point; and LPV GS at each bound parameter

\begin{tabular}{|c|c|c|c|c|}
\hline \multicolumn{3}{|c|}{ Classical GS (multi-model) } & \multicolumn{2}{|c|}{ LPV GS } \\
\hline $\begin{array}{c}\text { Operating } \\
\text { point } j\end{array}$ & $K_{p}$ & $K_{I}$ & $K_{p}$ & $K_{I}$ \\
\hline 1 & 0.97 & $4.9210^{-3}$ & \multirow{2}{*}{$\left(T_{\max }\right): 0.31$} & \multirow{2}{*}{$\left(T_{\max }\right): 1.3410^{-3}$} \\
\hline 2 & 0.53 & $3.8210^{-3}$ & & \\
\hline 3 & 0.37 & $3.4110^{-3}$ & \multirow{2}{*}{$\left(T_{\min }\right): 0.15$} & \multirow{2}{*}{$\left(T_{\min }\right): 6.9710^{-4}$} \\
\hline 4 & 0.14 & $2.8610^{-3}$ & & \\
\hline
\end{tabular}
space.

Table 2. PI parameter values
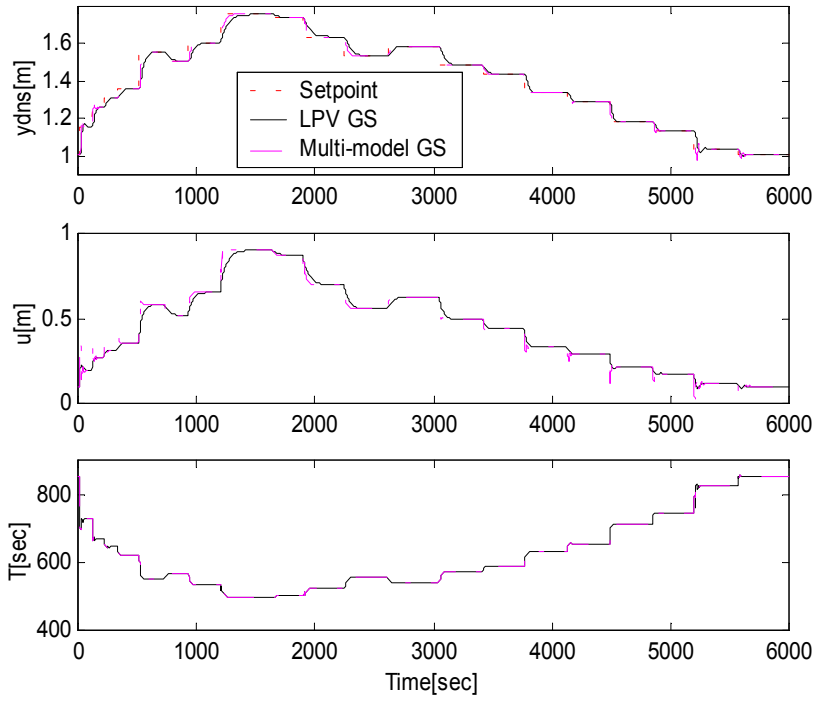

Fig.5. Closed-loop simulation results for all operation range and evolution of varying parameter $T$.

The LPV GS law is obtained using the algorithm based on the computation of polytopic coordinates described in Section 2.

The PI parameter values obtained by both methods are shown in Table 2 and Fig.4. In these figure it is 
shown the PI values obtained by multi-model GS and by LPV model. It can be observed than these values are practically equal than the parameter values obtained by multi-model GS because the LPV canal model and multi-model are very similar.

In Fig.5 the closed-loop results using both PI controllers are represented. This test study is relevant because the parameter $T$ varies along of the all operating range. It can be observed that the multimodel GS provide results less conservative than LPV GS, i.e. step responses faster (less bandwidth).

Besides for little gain openings we can see that this former technique provides large overshoot in comparison with the last technique due to two main reasons: is less conservative and a zero is incorporated in the closed-loop.

Both methodologies provide stability results for any parameter variation. But, while LPV GS assures this property just when the controller is computed in the multi-model GS the quadratic stability of closed-loop is checked after of calculating the controller by stability theorem, using LMIs (Tanaka,2001).

\section{CONCLUSIONS}

In this paper a comparative study of classical multimodel GS and LPV GS for an irrigation canal is carried out considering PI controllers. They are widely used in canal control because higher order controllers are numerically more fragile and computational demanding. The main conclusions provided by this work are:

- The LPV GS produces more conservative results than conventional GS. This is caused by the assumption that the parameters vary arbitrarily fast, the resulting synthesis inequalities become too large. Then, the accuracy and the potentiality of LPV models are not useful through advanced control methods used for this technique.

- The LPV GS design the controller assure directly the stability and performance for any parameter variation inside the parameter space. On the other hand, the global stability of multi-model GS is guaranteed after calculating controller values. In this case if the quadratic stability condition is not fulfilled, it is repeated the computation of the controller with more relaxed control objectives.

- The LPV model can be used in both LPV and conventional GS. Notice that controller values obtained by conventional GS techniques are practically equal using LPV model and multi-model.

\section{REFERENCES}

Apkarian, P., P. Gahinet and G. Becker (1995). "Selfscheduled $H_{\infty}$ control of linear parameter varying systems: A Design Example", Automatica, vol.31(9), pp.1251-1261.
Åström, K.J. and B. Wittenmark (1997). Computer Controlled Systems: Theory and Design, $3^{\text {rd }}$ Ed., Prentice-Hall.

Bamieh, B. and L.Giarré (2002). "Identification of linear parameter varying models", International Journal of Robust and Nonlinear Control.

Becker, G. and A. Packard (1994). "Robust performance of linear parametrically varying systems using parametrically-dependent linear feedback", System and Control Letters, vol.23, pp.205-215.

Bolea, Y., V.Puig., J.Blesa, M.Gómez and J.Rodellar (2002). "Identification of an open canal: parametric black-box vs. Muskingum approaches", IEEE Int. Conf. on Systems, Man and Cybernetics, vol.5.

Bolea, Y, V. Puig, J.Blesa, M.Gómez and J. Rodellar (2004). "An LPV model for canal control", 10 th IEEE Int. Conf. on Methods and Models in Automation and Robotics, MMAR'04, Poland.

Bolea,Y. and V.Puig (2005). "Gain scheduled PI(D) controllers for linear parameter with time delay", submitted in Control Engineering Practice.

Chentouf, B., C.Z. Xu and M. Boulbrachene (2001). "Robust regulation of a river reach governed by Hayami model", Proceedings of the $40^{\text {th }}$ IEEE Control and Decision Conference, vol.5, pp: 4962-4967.

Gómez, M., J. Rodellar, and J.A. Mantecon. (2002). "Predictive control method for decentralized operation of irrigation canals", Appl. Math. Modelling, vol.26, pp.1039-1056.

Goto, H.; K., S. Takeyasu, S. Masuda, and T. Amemiya (2003). "A gain-scheduled model predictive control for linear-parameter-varying max-plus-linear systems", American Control Conference, vol.5, pp.4016-4021.

Groot Wassink, M., M. van de Wal, C. Scherer and O. Bosgra (2005). "LPV control for a wafer stage:beyond the theoretical solution", Control Enginnering Practice, vol.13, pp.231-245.

Johansen, T.A. and R. Murray-Smith ed. (1996).“The Operating Regime to Nonlinear Modelling and Control", Multiple Model Approaches Modelling and Control, Taylor\&Francis.

Ljung, L. (1999). System Identification-Theory for the User ( $2^{\text {nd }}$ Edition). Prentice Hall, Englewood Cliffs, NJ.

Packard, A. and M. Kantner (1996). "Gain scheduling the LPV way", Proc. of $35^{\text {th }}$ IEEE Control and Decision Conference (CDC'96), pp.3938-3941.

Rugh, W.J. and J.S. Shamma (2000). "Research on Gain Scheduling", Automatica, vol.36, pp.1401-1425.

Schuurmans, J., O.H. Brosga and R. Brouwer (1995). "Openchannel flow model approximation for controller design”, Appl. Math. Modelling, vol.19, pp.525-530.

Shamma, J.S. and M. Athans (1990). "Analysis of gain scheduled control for nonlinear plants", IEEE Trans. Automatic Control, vol.35(8), pp.898-907.

Shamma, J.S., and M. Athans (1991). "Guaranteed Properties of Gain Scheduled Control for Linear Parameter Varying Plants", Automatica, vol.27, pp.559564.

Tanaka, K and , H.O. Wang (2002). Fuzzy control systems design and analysis. A linear matrix inequality approach, John Wiley \& Sons.

Wu, F. and A. Packard (1995). "LQG Control Design for LPV Systems", American Control Conference, pp.998994.

Yu, Z., H.Chen and P.-Y. Woo (2002). "Gain-scheduled LPV $H_{\infty}$ control based on LMI approach for a robotic manipulator", Journal of Robotic Systems, vol.19(2), pp.585-593. 